Bangladesh J. Bot. 36(1): 53-59, 2007 (June)

\title{
NEW RECORDS OF PHYTOPLANKTON FOR BANGLADESH. 2. CRYPTOPHYCEAE AND SYNUROPHYCEAE
}

\author{
Moniruzzaman Khondker", Rauf Ahmed Bhuiyan, Jenat Yeasmin, \\ Munirul Alam ${ }^{1}$, R. Bradley SaCk ${ }^{2}$, Anwar Huq ${ }^{3}$ and Rita R. Colwell ${ }^{2,3,4}$ \\ Department of Botany, University of Dhaka, Dhaka-1000, Bangladesh
}

Key words: Phytoplankton, New records, Cryptomonads, Synuroids, Ponds

\begin{abstract}
This study presents two species of Rhodomonas, four species of Chroomonas, six species of Cryptomonas and Cryptochrysis minor, Cyanomonas coeruleus, Chrysodidymus synuroideus and Mallomonas akrokomos. These species have been reported from some ponds of Mathbaria in Pirojpur and Bakerganj of Barisal district in Bangladesh.
\end{abstract}

\section{Introduction}

Cryptomonads are the most frequent form of micro-algae found in the communities of freshwater micro-phytoplankton (Islam and Khondker 1993, 1997). The group is represented worldwide by about 63 species, five varieties and two formae (Huber-Pestalozzi 1968). In Bangladesh, three species of this group namely, Rhodomonas lacustris Pascher, Cryptomonas ovata Ehr. and C. reflexa var. recurva (Marsson) Skuja have so far been reported (Islam and Khondker 1993, 1997). On the other hand, study on the members of scaly chrysophytes (i.e., class Synurophyceae) drew less attention because of their complex body structure. Body surface of these algae is ornamented with variously arranged siliceous scales, plates and bristles which require electron microscopy and are of tremendous taxonomic value. However, Takahashi and Hayakawa (1979) reported ten species (samples collected from Bangladesh but scanned via electron microscope in Japan) of the class Synurophyceae from Bangladesh. Recently Aziz (2000) reported Synura uvella Ehr. from an Azolla production pond near Dhaka. He also reported Mallomonas sp. from some northern districts of Bangladesh (Aziz and Tanbir 2003).

Cryptomonads and scaly chrysophytes have been frequently encountered in the plankton samples collected from different ponds of Mathbaria of Pirojpur district and Bakerganj of Barisal district in between 2004 and 2006 under a multidisciplinary multi-year National Institute of Health (NIH, USA) funded research project. The present paper deals with an illustrated account of the species belonging to Cryptophyceae and Synurophyceae.

\section{Materials and Methods}

Samples of phytoplankton were collected in a concentrated form from eight and six permanent stations of Bakerganj and Mathbaria, respectively in between 2004 and 2006 (Khondker et al. 2006). All the investigated water bodies were ponds except one river channel (Station No. 5, Bakerganj). Details of the methodologies have been described in Khondker et al. (2006).

*Corresponding author. E-mail: <khondker56@yahoo.com>. ${ }^{1}$ International Center for Diarrhoeal Disease Research, Bangladesh, Dhaka, Bangladesh. ${ }^{2}$ Johns Hopkins Bloomberg School of Public Health, Baltimore, Maryland. ${ }^{3}$ Center of Marine Biotechnology, University of Maryland Biotechnology Institute, Baltimore, Maryland. ${ }^{4}$ University of Maryland Institute for Advanced Computer Studies, College Park, Maryland, USA. 


\section{Results and Discussion}

In the present investigation 14 species of Cryptophyceae and two species of Synurophyceae were identified from the pelagic plankton communities of different ponds of Mathbaria and Bakerganj. An illustrated account of these species is presented in this paper. For the systematic arrangement, Huber-Pestalozzi (1968) and Kristiansen and Preisig (2001) have been followed.

\section{Division: Pyrrhophyta; Class: Cryptophyceae Family: Cryptochrysidaceae}

1. Cryptochrysis minor Nygaard

(Fig. 6)

(Huber-Pestalozzi 1968, fig. 5A, p. 18)

Cells ellipsoidal, small, free-swimming, both ends equally and regularly rounded, anterior end convex which is asymmetrically bilobed, posterior end narrow and blunt at the pole; pyrenoid present, dorsal in position; cells $11 \mu \mathrm{m}$ long and $6 \mu \mathrm{m}$ broad, flagella two, slightly unequal, longest one $7 \mu \mathrm{m}$ long.

Mathbaria, Station No. 6, 22.06.2004.

\section{Family: Cryptomonadaceae}

\section{Rhodomonas minuta Skuja}

(Huber-Pestalozzi 1968, Pl. 2, fig. 9, p. 23)

(Figs. 16 a-d, 17)

Cells more or less conical, posterior end blunt, little or sharply pointed; chromatophore discoid, pyrenoid single, olive brown or dark red or blue-green in colour; cells 9-15 $\mu \mathrm{m}$ long and 5 - $8 \mu \mathrm{m}$ broad; flagella two, unequal, very thin, one is near to body length, the other one little shorter.

Bakerganj, Station No. 1, 7, 8; 12.07.2004, 06.09.2004. Mathbaria, Station No. 1, 2; 22. 06 2004, 16.08.04, 30.08.2004.

\section{Rhodomonas minuta var. nannoplanktica Skuja}

(Figs. 15 a-b) (Huber-Pestalozzi 1968, Pl. 2, fig. 10, p. 23)

Cells more or less conical, posterior end blunt; chromatophore one and olive to brown or red in colour, pyrenoid single; cells 6 - $7 \mu \mathrm{m}$ long, 3 - $4 \mu \mathrm{m}$ broad, flagella two, unequal, 7 - $12 \mu \mathrm{m}$ long. The variety differs from the type mainly by having smaller dimension.

Mathbaria, Station No. 4, 16.08.2004; Bakerganj, Station No. 3, 29.11.2004.

4. Chroomonas acuta Utermöhl

(Figs. 1 a-d, 2)

(Huber-Pestalozzi 1968, Pl. 3, fig. 19, p. 33)

Cells compressed, anterior end truncate, posterior end gradually narrowed, tip rounded, broader towards the anterior end which is asymmetrically bilobed; chromatophore single, olive to dull-green, pyrenoid prominent; cells 7 - $11 \mu \mathrm{m}$ long, 4 - $6 \mu \mathrm{m}$ broad, flagella 5-10 $\mu \mathrm{m}$ long.

Mathbaria, Station No. 1, 2; 30.08.2004; 16.08.2004; Bakerganj, Station No. 3, 29.11.2004.

5. Chroomonas coerulea (Geitl.) Skuja

(Figs. 3a-b)

(Huber-Pestalozzi 1968, Pl. 3, fig. 12a, p. 27)

Cells ellipsoidal, broader towards the anterior end which is asymmetrically bilobed, narrowed posteriorly with broadly rounded tip; $6-7 \mu \mathrm{m}$ long, $4.5-5.5 \mu \mathrm{m}$ broad.

Mathbaria, Station No. 4, 16.08.2004. 
6. Chroomonas nordstedtii Hansgirg

(Figs. 4 a-b)

(Huber-Pestalozzi 1968, Pl. 3, fig. 13, p. 28)

Cells ellipsoidal or elongate ovoid, broader towards the anterior end which is asymmetrically bilobed, narrowed posteriorly with broadly rounded pole; chromatophores two, broad, parietal bands, pyrenoid present; cells $16.0-17.8 \mu \mathrm{m}$ long and $8.0-10.2 \mu \mathrm{m}$ broad, flagella $5-17 \mu \mathrm{m}$ long.

Mathbaria, Station No. 4, 06.08.2004, 04.07.2005.
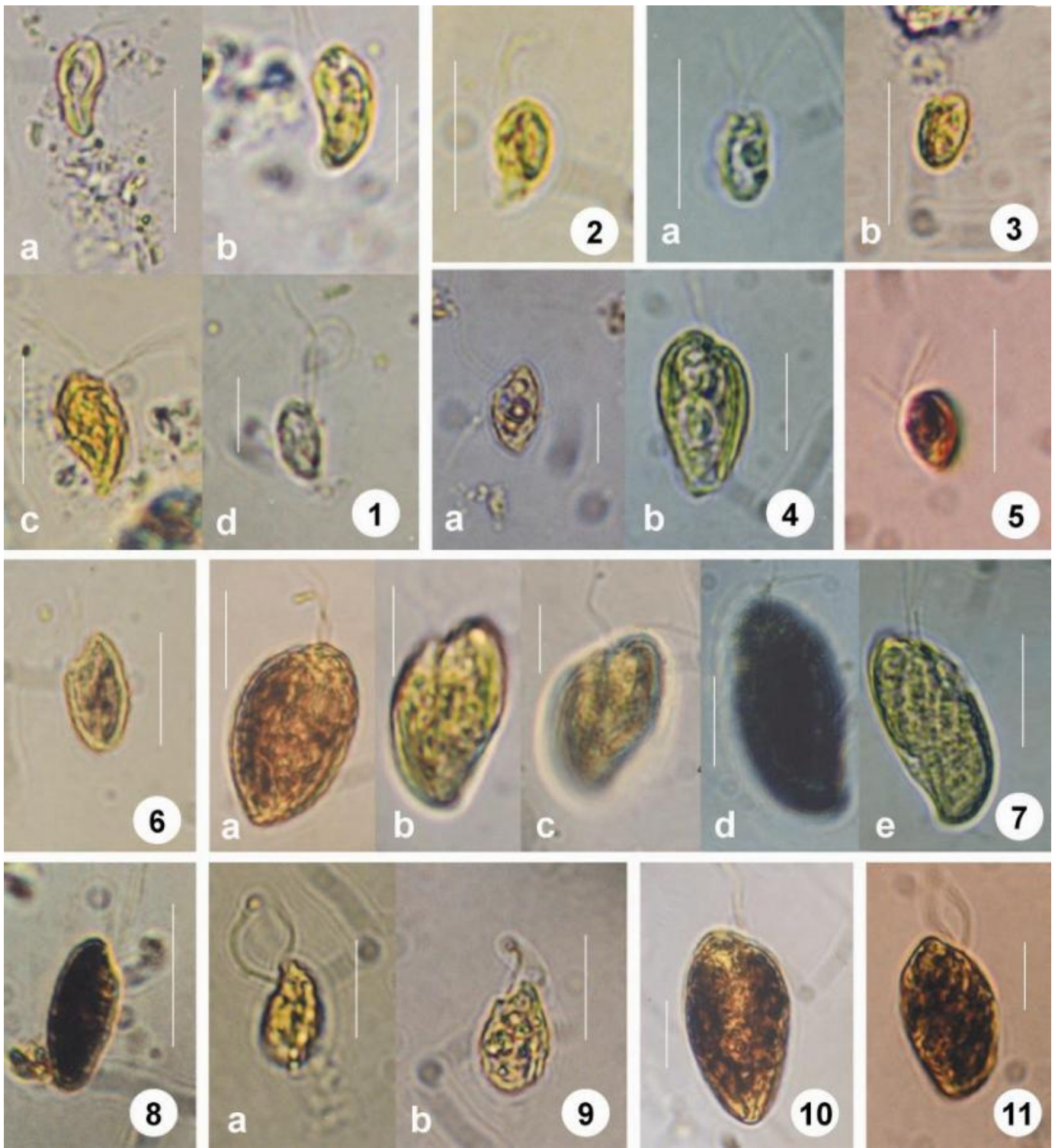

Figs. 1-11. 1 a-d, 2. Chroomonas acuta, 3 a-b. C. coerulea, 4 a-b. C. nordstedtii, 5. C. nordstedtii var. minor, 6. Cryptochrysis minor, 7 a-e. Cry. erosa, 8. Cry. lucens 9 a-b. Cyanomonas coeruleus, 10,11. Cryptomonas obovata. $($ Bars $=10 \mu \mathrm{m})$. 
7. Chroomonas nordstedtii fa. minor Nygaard

(Huber-Pestalozzi 1968, fig. 141, p. 29).

Cells solitary, ovoid, small, both the ventral and dorsal margin convex. Cells $6 \mu \mathrm{m}$ long and 4 $\mu \mathrm{m}$ broad, flagella two, unequal, inserted sub-apically; small flagellum $3 \mu \mathrm{m}$ long and larger flagellum $5 \mu \mathrm{m}$ long.

The forma differs from that of the type by being smaller in size.

Bakerganj, Station and sample No. 8, 29.03.2004; 12.07.2004.

8. Cyanomonas coeruleus Lackey

(Figs. 9a-b)

(Smith, 1952, p. 631; Edmondson, 1959, fig. 6.407, p.164)

Cells broadly ovate or ellipsoidal, posterior end not pointed, broadly rounded; chromatophores several and blue-green in colour; cells $10 \mu \mathrm{m}$ long and $7 \mu \mathrm{m}$ broad; flagella two, inserted in an anteriorly placed sub-lateral groove.

Mathbaria, Station No. 1, Collection date: 30.08.2004.

9. Cryptomonas erosa Ehr.

(Figs. 7a-e)

(Huber-Pestalozzi 1968, fig. 28, p. 51).

Cells broadly ovate or ellipsoidal with the left hand margin more convex, apex of the cell evenly bilobed, deep apical depression present, gullet broad, breadth is $1 / 2$ less than the length; two elongate parietal chromatophore present; brown, blue or reddish in colour; cells 20 - $30 \mu \mathrm{m}$ long; $10-15 \mu \mathrm{m}$ broad, flagella two, equal or unequal, short flagellum $8 \mu \mathrm{m}$ and long flagellum $15 \mu \mathrm{m}$ in length.

Mathbaria, Station No. 6, 16.08.2004; Bakerganj, Station No. 1, 8; 12.07.2004, 06.09.2004.

10. Cryptomonas lucens Skuja

(Fig. 8)

(Huber-Pestalozzi 1968, Pl. 6, fig. 37, p. 58).

Cells broadly ovate or ellipsoidal, apex of the cell evenly bilobed, deep apical depression present, gullet broad, breadth $1 / 2$ or more than the length; two elongate parietal chromatophore present; cells $11 \mu \mathrm{m}$ long; $7 \mu \mathrm{m}$ broad, flagella two, unequal; longer one $10 \mu \mathrm{m}$ and shorter one 7 $\mu \mathrm{m}$ long.

Bakerganj, Station No. 6, 29.11.2004.

11. Cryptomonas marssonii (?) Skuja

(Figs. 12 a-b)

(Huber-Pestalozzi 1968, Pl. 4, fig. 37, p. 58).

Cells broadly ovate or ellipsoidal, apex of the cell evenly bilobed, gullet broad, breadth $1 / 2$ or more than the length; chromatophore two, elongate and parietal; cells $26 \mu \mathrm{m}$ long; $11 \mu \mathrm{m}$ broad, flagella two, equal or unequal, $11 \mu \mathrm{m}$ long.

Notes: The shape of the present taxon resembles to some extent with Chroomonas caudata (HuberPestalozzi, 1.c., pl. 3, fig. 20) but the cell dimension is just half of the present taxon. In terms of cell dimension the present taxon fits well with Cryptomonas marsonii but the cell shape is slightly different. So, the present taxon has been tentatively placed under Cryptomonas marsonii.

Bakerganj, Station No. 8, 06.09.2004.

12. Cryptomonas obovata Skuja

(Figs. 10-11)

(Huber-Pestalozzi 1968, pl. 5, fig. 27, p. 51).

Cells broadly ovate or ellipsoidal, evenly bilobed apex, gullet broad, breadth $1 / 2$ or more than the length; chromatophore two, elongate and parietal; cells $25 \mu \mathrm{m}$ long; $13 \mu \mathrm{m}$ broad, flagella two, equal or unequal; $12 \mu \mathrm{m}$ long.

Bakerganj, Station No. 1, 12.07.2004. 
13. Cryptomonas phaseolus Skuja

(Fig. 14)

(Huber-Pestalozzi 1968, Pl. 5, fig. 26, p. 50)

Cells elliptical, both the ends rounded, periplast thin, hyaline, chromatophore two, spreaded throughout the body length, partly overlapped, violet-brownish, pyrenoid absent; $13 \mu \mathrm{m}$ long and $7 \mu \mathrm{m}$ broad; flagella two, somewhat unequal, longer one $15 \mu \mathrm{m}$ and shorter one $9 \mu \mathrm{m}$ long.

Mathbaria, Station No. 1, 01.02.2005.

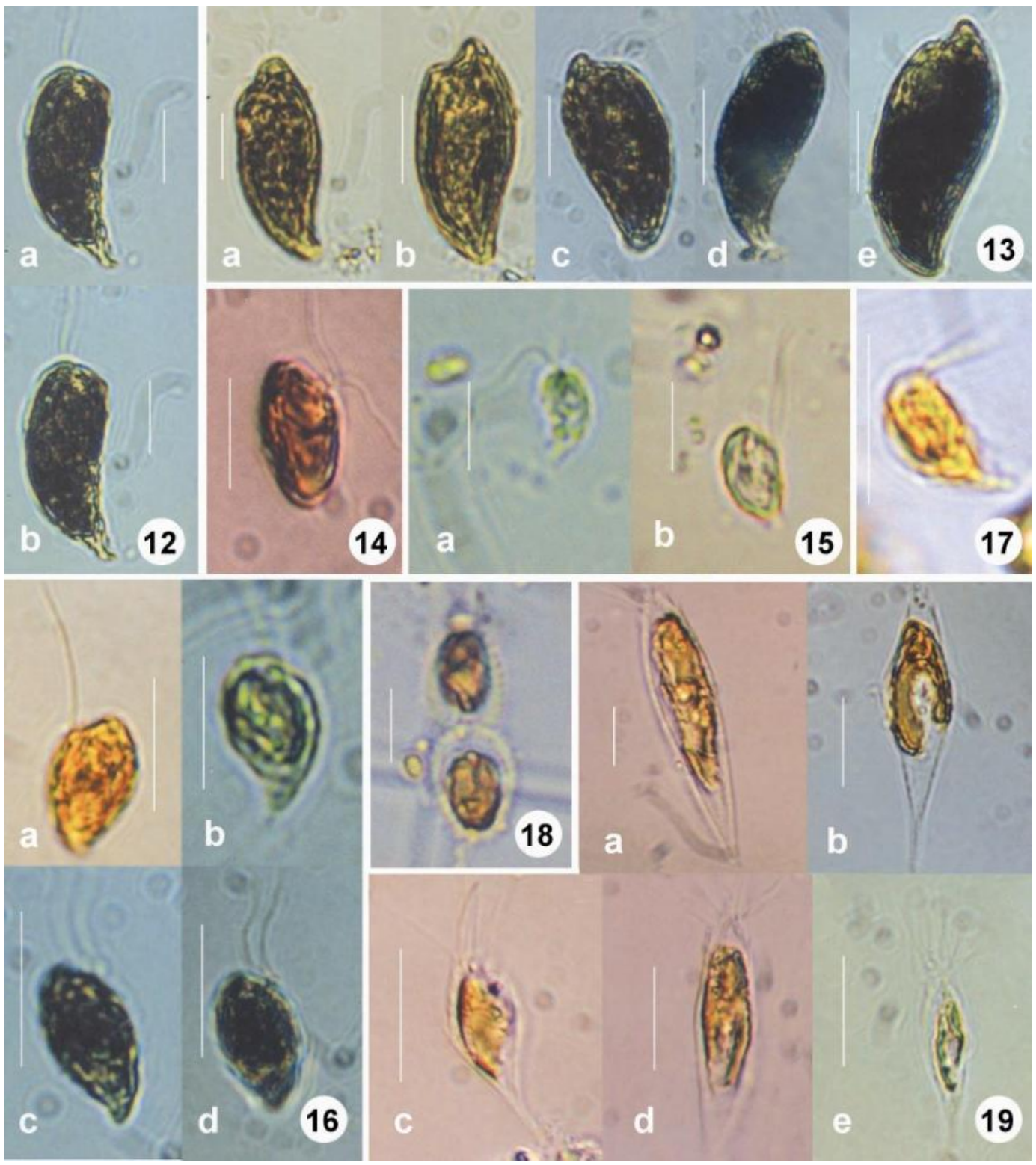

Figs. 12-19. 12 a-b. Cryptomonas marssonii (?), 13 a-e. Cry. reflexa, 14. Cry. phaseolus, 15 a-b. Rhodomonas minuta var. nannoplanktica, 16 a-d, 17. R. minuta, 18. Chrysodidymus synuroideus, 19 a-e. Mallomonas akrokomos. (Bars $=10 \mu \mathrm{m})$. 
14. Cryptomonas reflexa Skuja

(Figs. 13 a-e)

(Huber-Pestalozzi 1968, fig. 40, p. 59).

Cells relatively bigger, broadly ovate or ellipsoidal, spindle shaped, anterior end curved, posterior end pointed, gullet broad, breadth $1 / 2$ or more than the length; pyrenoid one, chromatophore two, elongate and parietal; cells 27 - $37 \mu \mathrm{m}$ long; 12 - $16 \mu \mathrm{m}$ broad, flagella two, equal or unequal; 7 - $10 \mu \mathrm{m}$ long.

Bakerganj, Station No. 3, 6; 06.09.2004.

\section{Division: Chrysophyta; Class: Synurophyceae Family: Mallomonadaceae}

15. Mallomonas akrokomos Ruttner

(Figs. 19 a-e)

(Edmondson 1959, p. 154, fig. 6.332)

Cells solitary having siliceous envelope, bristles long, terminally located like the bristles of a brush, covering the body. Each cell contains a bilobed massive chloroplast without stigma; cells 17 - $42 \mu \mathrm{m}$ long and 6 - $10 \mu \mathrm{m}$ broad, chromoplastid 12 - $18 \mu \mathrm{m}$ long and $6 \mu \mathrm{m}$ broad; bristles 8 $15 \mu \mathrm{m}$ long.

Mathbaria, Station No. 2, 03.01.2005, 01.02.2005; Bakerganj, Station No. 3, 29.11.2004.

\section{Family: Synuraceae}

16. Chrysodidymus synuroideus Prowse

(Figs. 18)

(Kristensen and Preisig 2001, p. 71, f.50).

Colony two celled, cell shape varies a little, cells having an armour of siliceous scales, consisting of a perforated oval plate with a short apical spine; chloroplast two, pyrenoid absent; larger cell $15.5 \mu \mathrm{m}$ long and $10.5 \mu \mathrm{m}$ broad; smaller cell $12.0 \mu \mathrm{m}$ long and $10.5 \mu \mathrm{m}$ broad.

Mathbaria, Station No. 5, 20.06.2005; Mathbaria, Station No. 2, 03.01.2005; Bakerganj, Station No. 3, 29.11.2004.

\section{Acknowledgements}

The research as an integral part of the major multidisciplinary project entitled 'Epidemiology and Ecology of Vibrio cholerae in Bangladesh' was financed by the National Institute of Health (NIH) research grant \# 1RO1A13912901 under the collaborative agreement between the International Centre for Diarrhoeal Disease Research, Bangladesh (ICDDR,B) and Johns Hopkins Bloomberg School of Public Health. The authors gratefully acknowledge the NIH ecological surveillance team at ICDDR,B for kindly supporting this research.

\section{References}

Aziz, A. 2000. Synura uvella Ehr. (Chrysophyceae): A new record for Bangladesh. Bangladesh J. Bot. 29(1): 79-80.

Aziz, A. and M. Tanbir. 2003. Algal flora of some northern districts of Bangladesh. Bangladesh J. Plant Taxon. 10(1): 63-77.

Edmondson, W.T. (Ed.). 1959. Freshwater biology. John Wiley \& Sons, Inc. pp. 1248.

Huber-Pestalozzi, G.H. 1968. Das Phytoplankton des Süsswassers. Systematik und Biologie. 3. Teil: Cryptophyceae, Chloromonadophyceae, Dinophyceae. E. Schweizerbart'sche Verlagsbuchhandlung (Nägele u. Obermiller), Stuttgart, Germany. pp. 322.

Islam, A.K.M.N. and M. Khondker. 1993. Some unicellular flagellate algae of Bangladesh. J. Asiat. Soc. Bangladesh, Sci. 19:103-108. 
Islam, A.K.M.N. and M. Khondker. 1997. New records of some flagellate algae for Bangladesh-5, Chlamydomonas, Pascherina, Pyrobotrys, Cryptomonas and Chilomonas. Bangladesh J. Pl. Taxon. 4(2): 13-23.

Khondker, M., R.A. Bhuiyan, J. Yeasmin, M. Alam, R.B. Sack, A. Huq and R.R. Colwell. 2006. New records of phytoplankton for Bangladesh. 1. Cyanophyceae. Bangladesh J. Bot. 35(2): 173-179.

Kristiansen, J. and H.R. Preisig (Eds.). 2001. Encyclopedia of Chrysophyte genera. Bibliotheca Phycologica, Band 110. J. Cramer, Berlin, Germany. pp. 260.

Smith, G.M. 1952. The freshwater algae of the United States. McGraw-Hill Book Co., New York. pp. 719.

Takahashi, E. and T. Hayakawa. 1979. The Synuraceae (Chrysophyceae) in Bangladesh. Phykos 18(1\&2): 129-147.

(Manuscript received on 01 February, 2007; revised on 22 April, 2007) 